\title{
Pancreatic gangliocytic paraganglioma harboring lymph node metastasis: a case report and literature review
}

\author{
Keisuke Nonaka ${ }^{1,2}$, Yoko Matsuda ${ }^{1}$, Akira Okaniwa ${ }^{3}$, Atsuko Kasajima ${ }^{2}$, Hironobu Sasano ${ }^{2}$ and Tomio Arai ${ }^{*}$
}

\begin{abstract}
Background: Gangliocytic paraganglioma (GP) is a rare neuroendocrine neoplasm, which occurs mostly in the periampullary portion of the duodenum; the majority of the reported cases of duodenal GP has been of benign nature with a low incidence of regional lymph node metastasis. GP arising from the pancreas is extremely rare. To date, only three cases have been reported and its clinical characteristics are largely unknown.

Case presentation: A nodule located in the pancreatic head was incidentally detected in an asymptomatic 68-year-old woman. Computed tomography revealed 18-, 8-, and 12-mm masses in the pancreatic head, the pancreatic tail, and the left adrenal gland, respectively. Subsequent genetic examination revealed an absence of mutations in the MEN1 and VHL genes. Macroscopically, the tumor located in the pancreatic head was $22 \mathrm{~mm}$ in size and displayed an ill-circumscribed margin along with yellowish-white color. Microscopically, it was composed of three cell components: epithelioid cells, ganglion-like cells, and spindle cells, which led to the diagnosis of GP. The tumor was accompanied by a peripancreatic lymph node metastasis. The tumor in the pancreatic tail was histologically classified as a neuroendocrine tumor (NET) G1 (grade 1, WHO 2010), whereas the tumor in the left adrenal gland was identified as an adrenocortical adenoma. The patient was disease-free at the 12-month follow-up examination.
\end{abstract}

Conclusions: Pancreatic GP is associated with a higher incidence of metastasis and larger tumor size than duodenal GPs, suggesting that the primary organ of GP is an important prognostic factor.

Keywords: Pancreas, Gangliocytic paraganglioma, Neuroendocrine tumor grade 1, Adrenocortical adenoma, Case report

\section{Background}

Since first report in 1957 [1], gangliocytic paragangliomas (GPs) have been found to occur mostly in the periampullary portion of the duodenum [2], although some have been observed in the jejunum [3], pylorus [4], esophagus [5], nasopharynx [6], ovary [7], and lung [8]. The pancreas is also an extremely rare site of GP; only three cases have been reported to date, making the clinical characteristics of pancreatic GP unclear [9-11]. GPs are typically associated with an extremely good prognosis, but patients harboring lymph node metastases have occasionally been reported [12]. Radical surgery with lymph node dissection is therefore the mainstay of curative treatment [13]. GPs consist of three characteristic

\footnotetext{
*Correspondence: arai@tmig.or.jp

'Department of Pathology, Tokyo Metropolitan Geriatric Hospital, 35-2

Sakae-cho, Itabashi-ku, Tokyo 173-0015, Japan

Full list of author information is available at the end of the article
}

tumor components: epithelioid, spindle, and ganglionlike cells [14]. Histopathologically, it can be challenging to distinguish pancreatic GPs from other pancreatic neoplasms with neuroendocrine differentiation, such as paragangliomas and neuroendocrine tumors, because of their low incidence, rare location, and similar morphological and immunohistochemical characteristics $[15,16]$. Their clinical presentation and treatment strategy, however, appear to differ $[11,17,18]$, making precise differentiation crucial for pathological diagnosis.

This report describes a patient with pancreatic GP harboring lymph node metastasis. The patient was also found to have a pancreatic neuroendocrine tumor (NET) G1 (grade 1, WHO 2010) and an adrenocortical adenoma. Herein, the clinical characteristics of pancreatic GP are reviewed, as well as the differential diagnosis of these tumors from other endocrine tumors. 


\section{Case presentation \\ Clinical history}

An asymptomatic, 68-year-old Japanese woman with no significant past medical history was found incidentally to have a low-echoic nodule in the pancreatic head via abdominal ultrasonography. No endocrine tumors or diseases were detected in any of her family members. Her father died of stomach cancer. She neither smoked nor drank alcohol. Her blood pressure $(127 / 69 \mathrm{mmHg})$ was well controlled via antihypertensive drugs. She was slightly obese (body mass index $=25.8 \mathrm{~kg} / \mathrm{m}^{2}$ ). Physical examinations did not show any abnormalities. Upper gastrointestinal endoscopy did not detect any lesions in the stomach or duodenum. Endoscopic ultrasonography revealed a $21.6 \mathrm{~mm}$ diameter, low-echoic nodule in the pancreatic head. No dilation of the biliary or pancreatic duct was detected. On contrast-enhanced computed tomography $(\mathrm{CT})$ scans, the above-described lesion was peripherally enhanced during the arterial phase (Fig. 1a) and was weakly and heterogeneously enhanced during the portal phase (Fig. 1b). In addition, CT scans identified an 8-mm mass in the pancreatic tail (Fig. 1c) with a well-defined border and marked enhancement, and a 12$\mathrm{mm}$ mass in the left adrenal gland with heterogeneous enhancement. On fluorine-18 fluorodeoxyglucose $\left({ }^{18} \mathrm{~F}-\right.$ FDG) positron emission tomography (PET) imaging, the mass in the pancreatic head demonstrated a high ${ }^{18}$ F-FDG uptake with a maximal standardized uptake value (SUVmax) of 4.8 (Fig. 1d), which implied a potential for malignancy. The masses in the pancreatic tail and the left adrenal gland exhibited negative ${ }^{18} \mathrm{~F}-\mathrm{FDG}$ uptake. All the laboratory data was within normal limits, including serum tumor markers, carcinoembryonic antigen (CEA), carbohydrate antigen 19-9 (CA 19-9), pancreatic cancer-associated antigen-2 (Dupan-2), s-pancreas-1 antigen (Span-1), pro-gastrin-releasing peptide (Pro-GRP), and neuron-specific enolase (NSE). Pancreatic endocrine hormones were also within normal limits. Needle biopsies from the pancreatic head and tail did not provide a sufficient amount of materials for a pathological diagnosis. The patient underwent pylorus-preserving pancreaticoduodenectomy with lymph node dissection, distal pancreatectomy, and left adrenalectomy.

\section{Materials and methods}

DNA was extracted from a peripheral blood sample and sequences corresponding to exons 2-10 of the MEN1 gene, exons 1-3 of the $V H L$ gene, and exon 16 of the RET gene were PCR amplified. The PCR products were directly sequenced using the BigDye Terminator v1.1 Cycle Sequencing Kit (Life Technologies, CA, USA), according to the manufacturer's instructions, and analyzed on an automated sequencer (3130xl Genetic Analyzer, Life Technologies).

\section{Pathological findings \\ Gangliocytic paraganglioma in the head of the pancreas}

Macroscopic examination revealed a poorly demarcated, solid, yellowish-white tumor, measuring $22 \times 22 \times 17 \mathrm{~mm}$, in the head of the pancreas (Fig. 2a). Microscopically, the tumor was composed of an admixture of three cell types: epithelioid cells, ganglion-like cells, and spindle cells (Fig. 2b). The epithelioid cells, the major component
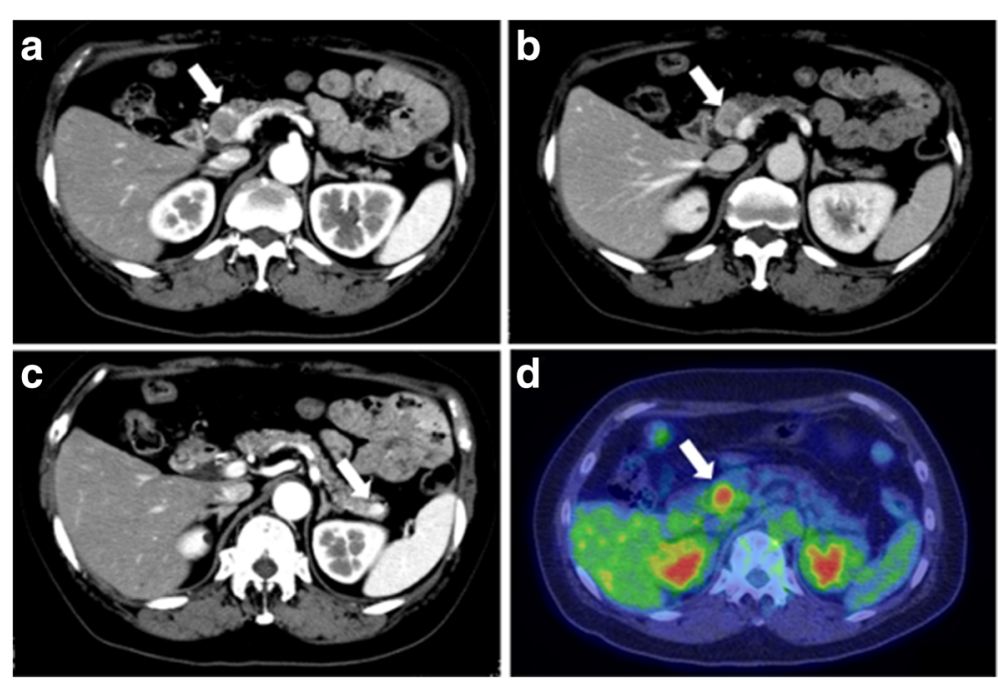

Fig. 1 Radiological images of masses in the pancreas and the left adrenal gland. a-c Contrast-enhanced computed tomography of the pancreas. a Arterial phase shows a mass with peripheral enhancement (arrow) in the pancreatic head. b Portal phase demonstrates a well-defined mass with weak and heterogeneous enhancement (arrow) in the pancreatic head. c In the pancreatic tail, arterial phase reveals a rounded and welldelineated mass with marked enhancement (arrow). $\mathbf{d}$ Fluorine-18 fluorodeoxyglucose positron emission tomography imaging shows a positive mass (arrow) in the pancreatic head 

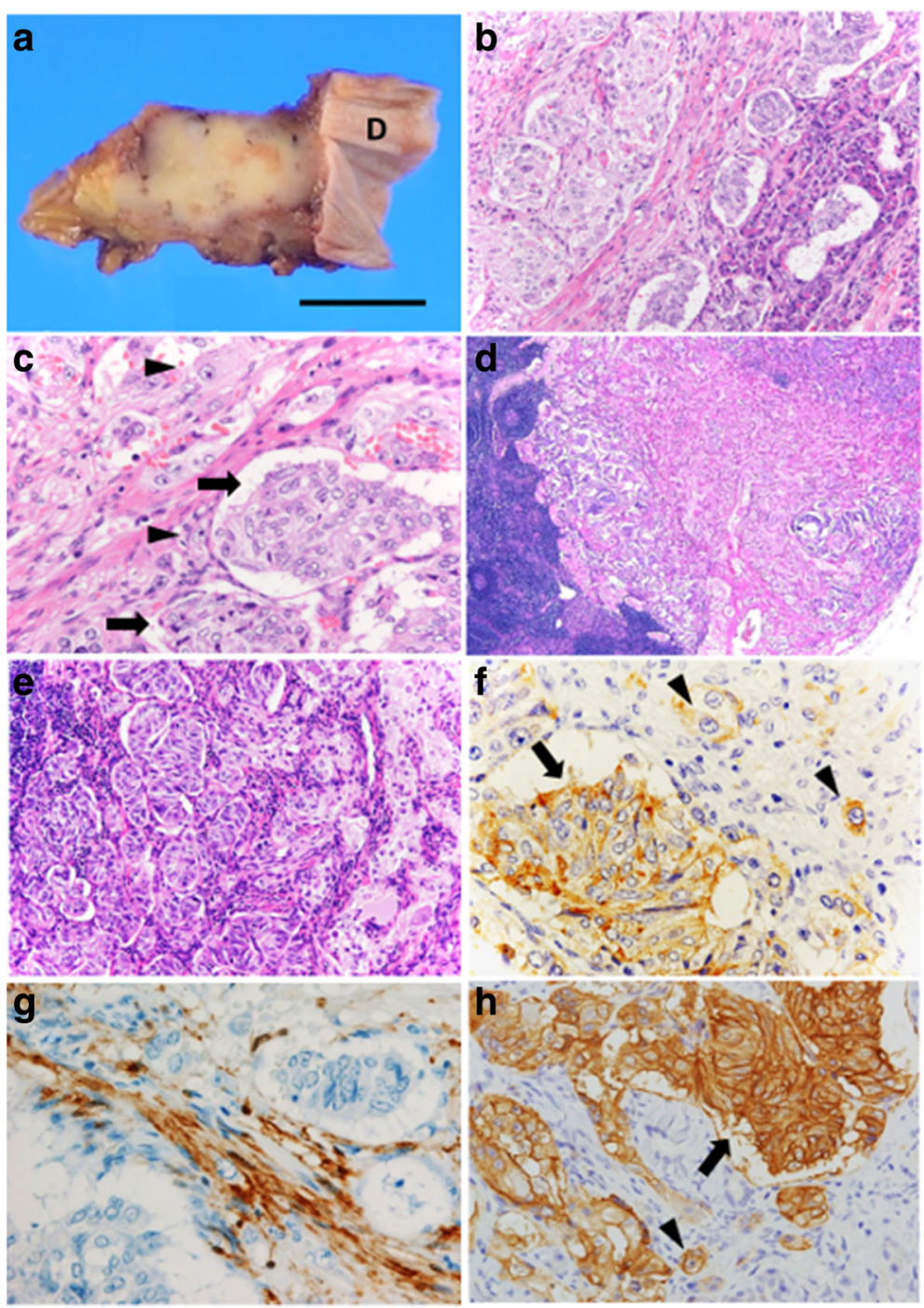

Fig. 2 Macroscopic, histological and immunohistochemical images of gangliocytic paraganglioma. a The cut surface of the pancreatic head shows a poorly marginated, yellowish-white tumor, surrounded by the pancreatic tissue without involving the duodenal wall (D) (scale bar $=1 \mathrm{~cm})$. $\mathbf{b}$ The tumor is seen adjacent to the pancreatic tissue (right side) (hematoxylin-eosin staining). c The tumor is composed of epithelioid cell nests (arrow), ganglion-like cells (arrowhead), and spindle cells (hematoxylin-eosin staining). $\mathbf{d}$ and $\mathbf{e}$ In the peripancreatic lymph node, the metastasizing lesion comprises all three cell types (hematoxylin-eosin staining, respectively). $\mathbf{f}$ The epithelioid (arrow) and ganglion-like cells (arrowhead) are positive for chromogranin A,

whereas the spindle cells are negative. $\mathbf{g}$ Only spindle cells are immunoreactive for S-100 protein. $\mathbf{h}$ SSTR2A is expressed in the epithelioid cells (arrow) and in a small number of the ganglion-like cells (arrowhead) with complete membranous positivity (score 3)

of the tumor, were rather uniform in size and formed small nests (Fig. 2c), exhibiting fine granular eosinophilic cytoplasm and round-to-ovoid nuclei with stippled chromatin. This component of the tumor showed vascular, perineural, and peripancreatic adipose tissue invasion. The ganglion-like cells, the smallest component of the tumor, had vesicular nuclei, conspicuous nucleoli, and an abundance of cytoplasm (Fig. 2c). The spindle cells exhibited thin, elongated, wavy-shaped nuclei resembling Schwann cells and were arranged in intertwining fascicles. Mitosis was not conspicuous, and foci of intratumoral necrosis were not observed. The surgical margins were negative for tumor cells. Tumor tissue, composed of all the three cell types, also showed metastasis to a peripancreatic lymph node (Fig. 2d, e).

The immunohistochemical findings of the three components are summarized in Table 1 . The epithelioid cells were positive for chromogranin A (Fig. 2f), synaptophysin, and pancytokeratin (AE1/AE3) but negative for catecholaminesynthesizing enzymes (tyrosine hydroxylase, dopamine $\beta$ hydroxylase, dopa decarboxylase, and phenylethanolamine $N$-methyltransferase). The ganglion-like cells were positive 
Table 1 Immunohistochemical findings obtained from the gangliocytic paraganglioma and the neuroendocrine tumor G1

\begin{tabular}{|c|c|c|c|c|}
\hline & \multicolumn{3}{|c|}{ GP in pancreatic head } & \multirow{2}{*}{$\begin{array}{l}\text { NET G1 in } \\
\text { pancreatic tail }\end{array}$} \\
\hline & $\bar{E}$ & $G$ & $\mathrm{~s}$ & \\
\hline Chromogranin A & + & + & - & + \\
\hline Synaptophysin & + & + & - & + \\
\hline S-100 protein & - & - & + & - \\
\hline Neurofilament & - & + & + & ND \\
\hline Pancytokeratin & + & + & - & ND \\
\hline SSTR1 & - & - & - & ND \\
\hline SSTR2A & + & + & - & ND \\
\hline SSTR3 & - & - & - & ND \\
\hline SSTR5 & - & - & - & ND \\
\hline Insulin & - & - & - & - \\
\hline Glucagon & - & - & - & + \\
\hline PP & - & - & - & - \\
\hline Serotonin & - & - & - & ND \\
\hline Somatostatin & + & + & - & - \\
\hline Tyrosine hydroxylase & - & - & - & ND \\
\hline Dopamine $\beta$-hydroxylase & - & - & - & ND \\
\hline Dopa decarboxylase & - & - & - & ND \\
\hline Phenylethanolamine $\mathrm{N}$-methyltransferase & - & + & - & ND \\
\hline PgR & + & + & - & + \\
\hline
\end{tabular}

GP gangliocytic paraganglioma, NET neuroendocrine tumor, G1 grade 1, E epithelioid cells, G ganglion-like cells, S spindle cells, SSTR somatostatin receptor, PP pancreatic polypeptide, $P g R$ Progesterone receptor, + positive, - negative; ND Not done

for synaptophysin and chromogranin A (Fig. 2f). Some of these cells were weakly positive for neurofilament, pancytokeratin, and phenylethanolamine $N$-methyltransferase. The spindle cells were positive for S-100 protein (Fig. 2g) and neurofilament. The Ki-67 labeling index (LI) of the tumor cells was $0.3 \%$, with no apparent difference among the three cell types. The immunohistochemical features of the metastatic lesion were identical to those of the primary tumor.

We also investigated the immunohistochemical expression of hormonal products and hormone receptors (Table 1). Somatostatin receptor (SSTR) 2A was expressed on the cell membranes of more than $50 \%$ of epithelioid cells (Fig. $2 \mathrm{~h}$ ), which was defined as a score of 3 [19]. SSTR2A expression was also detected in a small number of ganglion-like cells (Fig. 2h). The epithelioid cells and some ganglion-like cells were positive for progesterone receptor (PgR). All the three cell types were negative for pancreatic polypeptide (PP).

\section{Neuroendocrine tumor in the tail of the pancreas}

A well-demarcated, grayish-white tumor, $7 \times 7 \mathrm{~mm}$ in size was found in the pancreatic tail. Microscopically, the tumor was composed of round-to-columnar cells with uniformly round nuclei, a salt-and-pepper chromatin pattern, and an eosinophilic, finely granular cytoplasm, arranged in trabecular and ribbon-like patterns and accompanied by fibrous stroma (Fig. 3a, b). There was no evidence of mitotic figures, necrosis, or lymphovascular invasion. Immunohistochemical examination showed that the tumor cells were diffusely positive for chromogranin A and synaptophysin (Table 1). The Ki-67 LI was $0.5 \%$. Accordingly, the tumor was diagnosed as a pancreatic NET G1.

Table 1 also shows the results obtained from the immunohistochemical evaluation of hormonal products and hormone receptors. The tumor cells were diffusely positive for glucagon and PgR, but negative for PP.

\section{Adrenocortical adenoma in the left adrenal gland}

Macroscopic examination showed that the nodular lesion in the left adrenal gland was $15 \times 12 \mathrm{~mm}$ in size, well-circumscribed, and with a yellowish-tan coloring. Microscopically, the tumor was composed of both spongiocytic cells with a clear lipid-rich cytoplasm and lipid-poor compact cells with an eosinophilic cytoplasm (Fig. 3c, d). There was no evidence of mitosis, tumor necrosis, or vascular invasion. The pathological diagnosis was an adrenocortical adenoma. The surgical margins were negative for tumor cells. 


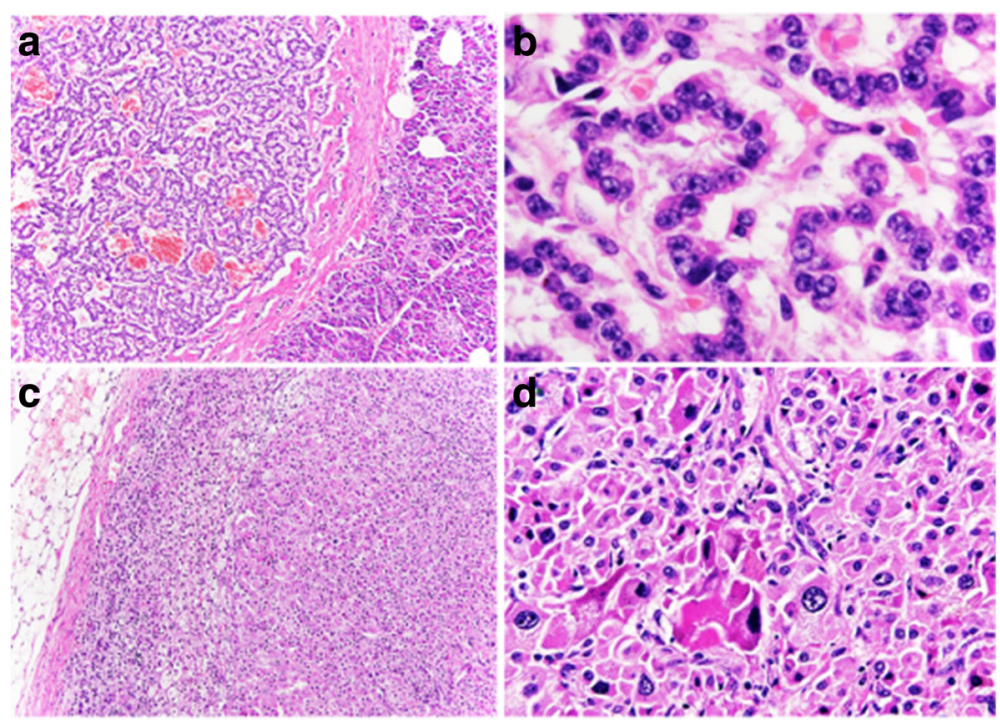

Fig. 3 Histological images of the tumor in the pancreatic tail and left adrenal gland. a The pancreatic tail tumor shows trabecular and ribbon-like growth patterns with fibrovascular stroma (hematoxylin-eosin staining). b The pancreatic tail tumor exhibits the following cytological features; small round nuclei, a salt-and-pepper chromatin pattern, and an eosinophilic, finely granular cytoplasm (hematoxylin-eosin staining). c The adrenal tumor is well-circumscribed and nonencapsulated (hematoxylin-eosin staining). $\mathbf{d}$ The adrenal tumor shows lipid-poor compact cells with an eosinophilic cytoplasm (hematoxylin-eosin staining)

\section{Gene mutation analysis}

DNA analysis of a peripheral blood sample showed no mutations in exons $2-10$ of the MEN1 gene, exons 1-3 of the $V H L$ gene, and exon 16 of the RET gene.

\section{Follow up}

The patient's postoperative course was uneventful. No signs of recurrence or metastasis were detected during a 1-year follow-up period.

\section{Discussion}

Table 2 summarizes the clinical features of the four patients with pancreatic GP, including our patient, identified to date [9-11]. The average age of these four patients was 63 years, and three (75\%) were women. Interestingly, the tumors in all four patients were located in the head of the pancreas, but our patient was the only patient without abdominal symptoms, such as abdominal pain and jaundice. Although duodenal GP is characterized by a favorable clinical course and a low incidence of lymph node metastasis (6.9\% of 173 patients [13]), with none showing distant metastasis, three $(75 \%)$ of the four patients with pancreatic GPs had metastases, including two with lymph node metastases and one with distant metastasis to the sternum. In addition pancreatic GPs were larger (mean diameter: $35 \mathrm{~mm}$ ) than duodenal GPs $(24.2 \mathrm{~mm})$. These results are consistent with the greater difficulty detecting early stage pancreatic than duodenal tumors. Furthermore, pancreatic GPs may have a higher potential for malignancy than duodenal GPs.

Pancreatic paraganglioma is important in the differential diagnosis of pancreatic GP, as the epithelioid cell component of GP is morphologically and immunohistochemically similar to paraganglioma $[15,16]$. Moreover, most pancreatic paragangliomas are nonfunctional and located in the pancreatic head [17]. To distinguish GP

Table 2 Summary of clinical features of pancreatic gangliocytic paraganglioma

\begin{tabular}{lllllllll}
\hline Reference & Year & Age (years) & Sex & Size $(\mathrm{mm})$ & Clinical Presentation & Metastasis & Operation & Outcome (months) \\
\hline Tomic et al. [11] & 1996 & 74 & Female & 40 & $\begin{array}{l}\text { Abdominal pain, Diarrhea, } \\
\text { Steatorrhea, Nausea, Vomiting, }\end{array}$ & (+) lymph node & PD & NER 20 \\
Henry et al. [10] & 2003 & 50 & Male & 25 & Cholestatic jaundice & (+) sternum & $\begin{array}{l}\text { PD, followed by } \\
\text { sternal resection }\end{array}$ & NER 18 from the \\
& & & & & & Fecond operation \\
Liu et al. [9] & 2008 & 60 & Female & 53 & Fever, Abdominal pain, Jaundice & NR & PD & NER 12 \\
Present case & 2016 & 68 & Female & 22 & None & (+) lymph node & PPPD & NER 12
\end{tabular}

$P D$ pancreaticoduodenectomy, $P P P D$ pylorus-preserving pancreaticoduodenectomy, NR not reported, NER no evidence of recurrence 
from paraganglioma, we therefore immunohistochemically stained the tumor in our patient for catecholaminesynthesizing enzymes and pancytokeratin (Table 1). Most paragangliomas, including the non-functioning type, are positive for catecholamine-synthesizing enzymes [20] but negative for pancytokeratin [15]. To date, the details of catecholamine-synthesizing enzyme expression in GP have not been studied. However, the epithelioid cell component of GP in our patient was negative for catecholamine-synthesizing enzyme expression, suggesting that the functionality of this cellular component of GP may differ from paraganglioma.

Expression of PgR and PP may differentiate "duodenal" GP from "duodenal" NET G1 [16]. Assessment of 12 duodenal GPs showed that the epithelioid cell component in 11 (91.7\%) were positive for PgR and PP. In contrast, duodenal NETs G1 were uniformly negative for PgR and PP expression. To date, no study has assessed the expression of PgR and PP in pancreatic GP. Interestingly, the immunohistochemical profile of $\mathrm{PgR}$ and $\mathrm{PP}$ expression in this patient were similar in the epithelioid cell component of the pancreatic GP and the NET, a result that may help in differentiating between pancreatic and duodenal GP. Further analysis is required to confirm the usefulness of PgR and PP expression in the diagnosis and differentiation of pancreatic GP.

Contrast-enhanced CT revealed differences in the contrast-enhanced patterns of GP in the pancreatic head and NET G1 in the pancreatic tail. FDG PET has been considered inadequate for the diagnosis of well-differentiated NETs due to their very slow growth [21]. The GP in this case, however, exhibited high ${ }^{18} \mathrm{~F}-\mathrm{FDG}$ uptake, indicating that ${ }^{18}$ F-FDG PET could be a practical tool for detecting pancreatic GPs or to differentiate pancreatic GPs from NETs.

Several somatostatin analogs (SAs), including octreotide, lanreotide, and pasireotide, have been shown to have therapeutic benefits in patients with advanced pancreatic NETs [18]. The effectiveness of SA therapy for patients with GPs is entirely unknown, as none of these patients to date has been treated with SA. The present study, along with a previously reported case of GP in the ampulla of Vater, showed that the epithelioid cell component was immunohistochemically positive for SSTR2A [22], suggesting that SA therapy may have benefit GPs. Further study is warranted.

The co-occurrence of three distinct endocrine tumors in one patient suggests the possibility of a genetic predisposition in this patient. The genetic and biological backgrounds of this peculiar combination of three different endocrine neoplasms remain largely unknown. Although we found nothing indicating an inherited condition, the patient should be carefully monitored to allow for the early detection of other endocrine-related neoplastic lesions.

\section{Conclusion}

This report describes the fourth reported case of pancreatic GP. Compared with duodenal GPs, pancreatic GPs were larger and had a higher incidence of metastasis, suggesting a greater potential for malignancy. Therefore, the primary organ of GP may be an important prognostic factor.

\begin{abstract}
Abbreviations
${ }^{18}$ F-FDG: Fluorine-18 fluorodeoxyglucose; CA 19-9: Carbohydrate antigen; CEA: Carcinoembryonic antigen; CT: Computed tomography; Dupan-

2: Pancreatic cancer-associated antigen-2; GP: Gangliocytic paragangliomas; LI: Labeling index; MEN 1: Multiple endocrine neoplasia type 1;

NET: Neuroendocrine tumor; NF 1: Neurofibromatosis type 1; NSE: Neuronspecific enolase; PCR: Polymerase chain reaction; PET: Positron emission tomography; PgR: Progesterone receptor; PP: Pancreatic polypeptide; ProGRP: Pro-gastrin-releasing peptide; SAs: Somatostatin analogues; Span-1: Spancreas-1 antigen; SSTR: Somatostatin receptor; SUVmax: Maximal

standardized uptake values; VHL: von Hippel-Lindau disease
\end{abstract}

\section{Acknowledgements}

We would like to thank the staff of the Department of Pathology, Kimitsu Chuo Hospital for preparing the histopathological slides.

Funding

There are no funding sources for this study.

Availability of data and materials

Not applicable.

\section{Authors' contributions}

KN conceptualized the case report and integrated the data; KN and YM performed the histopathologic evaluation and largely contributed to writing; AO provided clinical information; AK, HS, and TA carried out the histopathologic evaluation and approved the final manuscript. All authors read and approved the final manuscript.

\section{Ethics approval and consent to participate}

Ethical approval for this study was obtained from the Ethics Committee at the Kimitsu Chuo Hospital.

\section{Consent for publication}

Written informed consent was obtained from the patient for publication of the case report and any accompanying images. A copy of the written consent is available for review by the Editor-in-Chief of this journal.

\section{Competing interests}

The authors declare that they have no competing interests.

\section{Publisher's Note}

Springer Nature remains neutral with regard to jurisdictional claims in published maps and institutional affiliations.

\section{Author details}

'Department of Pathology, Tokyo Metropolitan Geriatric Hospital, 35-2 Sakae-cho, Itabashi-ku, Tokyo 173-0015, Japan. ${ }^{2}$ Department of Pathology, Tohoku University School of Medicine, 2-1 Seiryou-machi, Aoba-ku, Sendai-shi 980-8575, Japan. ${ }^{3}$ Department of Surgery, Kimitsu Chuo Hospital, 1010 Sakurai, Kisarazu, Chiba 292-8535, Japan.

Received: 26 January 2017 Accepted: 20 July 2017

Published online: 02 August 2017

\section{References}

1. Dahl EV, Waugh JM, Dahlin DC. Gastrointestinal ganglioneuromas; brief review with report of a duodenal ganglioneuroma. Am J Pathol. 1957;33: 953-65.

2. Sheahan K. Epithelial tumours of the small intestine. In: Shepherd NA, Warren BF, Williams GT, Greenson JK, Lauwers GY, Novelli MR, editors. 
Morson and Dawson's Gastrointestinal Pathology. London: Wiley-Blackwell; 2013. p. 422-41.

3. Aung W, Gallagher HJ, Joyce WP, Hayes DB, Leader M. Gastrointestinal haemorrhage from a jejunal gangliocytic paraganglioma. J Clin Pathol. 1995; 48:84-5.

4. Burke AP, Helwig EB. Gangliocytic paraganglioma. Am J Clin Pathol. 1989;92: $1-9$.

5. Weinrach DM, Wang KL, Blum MG, Yeldani AV, Laskin WB. Multifocal presentation of gangliocytic paraganglioma in the mediastinum and esophagus. Hum Pathol. 2004;35:1288-91.

6. Sinkre P, Lindberg G, Albores-Saavedra J. Nasopharyngeal gangliocytic paraganglioma. Arch Pathol Lab Med. 2001;125:1098-100.

7. Mahdavi A, Silberberg B, Malviya VK, Braunstein AH, Shapiro J. Gangliocytic paraganglioma arising from mature cystic teratoma of the ovary. Gynecol Oncol. 2003:90:482-5.

8. Hironaka M, Fukayama M, Takayashiki N, Saito K, Sohara Y, Funata N. Pulmonary gangliocytic paraganglioma: case report and comparative immunohistochemical study of related neuroendocrine neoplasms. Am J Surg Pathol. 2001;25:688-93.

9. Liu Y, Upadhyaya M, Song Q, Chen K. Pancreatic gangliocytic paraganglioma with thick tortuous drainage veins. Eur J Radiol Extra. 2009; 70:e116-8.

10. Henry C, Ghalel-Mechaoui H, Bottero N, Pardier T, Moindrot H. Gangliocytic paraganglioma of the pancreas with bone metastasis. Ann Chir. 2003;128: 336-8.

11. Tomic S, Warner T. Pancreatic somatostatin-secreting gangliocytic paraganglioma with lymph node metastases. Am J Gastroenterol. 1996;91:607-8.

12. Okubo Y, Yokose T, Tuchiya M, Mituda A, Wakayama M, Hasegawa C, et al. Duodenal gangliocytic paraganglioma showing lymph node metastasis: a rare case report. Diagn Pathol. 2010;5:27.

13. Okubo Y, Wakayama M, Nemoto T, Kitahara K, Nakayama H, Shibuya K, et al. Literature survey on epidemiology and pathology of gangliocytic paraganglioma. BMC Cancer. 2011;11:187.

14. Klöppel G, Arnold R, Capella C, Klimstra DS, Albores-Saavedra J, Solcia E, et al. Neuroendocrine neoplasms of the ampullary region. In: Bosman FT, Carneiro F, Hruban RH, Theise ND, editors. WHO Classification of Tumors of the Digestive System. Lyon: IARC press; 2010. p. 92-4

15. Ginesu GC, Barmina M, Paliogiannis P, Trombetta M, Cossu ML, Feo CF, et al. Nonfunctional paraganglioma of the head of the pancreas: A rare case report. Int J Surg Case Rep. 2016;28:81-4.

16. Okubo Y, Nemoto T, Wakayama M, Tochigi N, Shinozaki M, Ishiwatari T, et al. Gangliocytic paraganglioma: a multi-institutional retrospective study in Japan. BMC Cancer. 2015;15:269.

17. Liang W, Xu S. CT and MR Imaging Findings of Pancreatic Paragangliomas: A Case Report. Medicine (Baltimore). 2016;95:e2959.

18. Eriksson B. New drugs in neuroendocrine tumors: rising of new therapeutic philosophies? Curr Opin Oncol. 2010;22:381-6.

19. Volante M, Brizzi MP, Faggiano A, La Rosa S, Rapa I, Ferrero A, et al. Somatostatin receptor type $2 \mathrm{~A}$ immunohistochemistry in neuroendocrine tumors: a proposal of scoring system correlated with somatostatin receptor scintigraphy. Mod Pathol. 2007;20:1172-82.

20. Kimura N, Miura Y, Nagatsu I, Nagura H. Catecholamine synthesizing enzymes in 70 cases of functioning and non-functioning phaeochromocytoma and extra-adrenal paraganglioma. Virchows Arch A Pathol Anat Histopathol. 1992;421:25-32.

21. Adams S, Baum R, Rink T, Schumm-Drager PM, Usadel KH, Hor G. Limited value of fluorine-18 fluorodeoxyglucose positron emission tomography for the imaging of neuroendocrine tumours. Eur J Nucl Med. 1998;25:79-83.

22. Von Loga K, Konig A, Zustin J. Periampullary gangliocytic paraganglioma: a case report. In Vivo. 2010;24:321-4.

\section{Submit your next manuscript to BioMed Central and we will help you at every step:}

- We accept pre-submission inquiries

- Our selector tool helps you to find the most relevant journal

- We provide round the clock customer support

- Convenient online submission

- Thorough peer review

- Inclusion in PubMed and all major indexing services

- Maximum visibility for your research

Submit your manuscript at www.biomedcentral.com/submit
Biomed Central 